Revue d'histoire de l'Amérique française

ZWB REVUE D.HISTOIRE DE L'AMÉRIQUE FRANÇAISE

\title{
Les maladies de la misère - aspects de la santé publique à Montréal - 1880-1914
}

\section{Martin Tétreault}

Volume 36, numéro 4, mars 1983

URI : https://id.erudit.org/iderudit/304093ar

DOI : https://doi.org/10.7202/304093ar

Aller au sommaire du numéro

Éditeur(s)

Institut d'histoire de l'Amérique française

ISSN

0035-2357 (imprimé)

1492-1383 (numérique)

Découvrir la revue

Citer cet article

Tétreault, M. (1983). Les maladies de la misère — aspects de la santé publique à Montréal - 1880-1914. Revue d'histoire de l'Amérique française, 36(4), 507-526. https://doi.org/10.7202/304093ar d'utilisation que vous pouvez consulter en ligne.

https://apropos.erudit.org/fr/usagers/politique-dutilisation/ 


\title{
LES MALADIES DE LA MISÈRE ASPECTS DE LA SANTÉ PUBLIQUE Ä MONTRÉAL 1880-1914*
}

\author{
MARTIN TÉTREAULT, étudiant \\ Département d'histoire \\ Université de Montréal
}

Depuis bientôt plus d'une décennie les historiens se sont en quelque sorte rapprochés du corps humain; celui-ci est devenu l'objet d'une investigation historique au même titre que le pouvoir, l'échange ou les comportements sociaux. Des historiens, français et américains plus spécialement, ont compris que l'étude de tout ce qui pouvait s'apparenter à l'homme, pris dans sa corporalité propre, pouvait constituer un apport supplémentaire à la connaissance historique des sociétés ${ }^{1}$. En effet, l'étude de la santé d'une population, que ce soit par le biais de l'étude des institutions de santé, des groupes médicaux ou de l'épidémiologie, renvoie obligatoirement aux conditions de vie. Étudier l'état de santé d'une population c'est aussi se placer au carrefour d'avenues à la fois multiples et interdépendantes qui réfléchissent et surdéterminent certaines composantes de la vie sociale (conditions de travail et de logement, nature et qualité de l'alimentation, pour ne citer que les plus évidentes). Il va de soi que nous considérons le concept de santé selon son sens épidémiologique, c'est-à-dire comme phénomène de masse: c'est en vertu de ce dernier sens, on l'aura deviné, que l'étude de la santé se révèle intéressante pour l'histoire sociale.

Nous avons retenu l'exemple montréalais entre 1880 et 1914 pour diverses raisons: d'abord il s'agit d'une ville et, générale-

* Le présent texte constitue une version remaniée des chapitres III et IV de mon mémoire de maîtrise intitulé L'état de santé des Montréalais, 1880-1914, déposé au Département d'histoire de l'Université de Montréal en juillet 1979. Je remercie René Durocher, directeur de la recherche, Michel Brunet et Jacques Rouillard qui composaient le jury.

1 Entre autres parutions soulignons: Susan Reverby et David Rosner (dir.), Health Care in America. Essays in Social History (Philadelphie, Temple University Press, 1979); Todd L. Savitt, Medicine and Slavery. The Diseases and Health of Blacks in Antebellum America (Chicago, University of Illinois Press, 1978); Charles E. Rosenberg, The Cholera Years, The United States in 1832, 1846 and 1866 (Chicago, University of Chicago Press, 1962); James Harvey Young, The Toadstool Millionaires. A Social History of Patent Medicines in America... (Princeton, Princeton University Press, 1961); John S. Haller, American medicine in Transition, 1840-1910 (Chicago, University of Illinois Press, 1981); Richard E. Brown, Rockfeller Medicine men Medicine and Capitalism in America (Berkeley, University of California Press, 1979); ainsi que Judith Leavitt et Ronald Numbers (dir.), Sickness and Health in America Readings in the History of Medicine and Public Health (Madison, University of Wisconsin Press, 1978). 
ment, les problèmes de santé publique s'y posent avec beaucoup plus d'acuité qu'en milieu rural (spécialement en raison du risque plus élevé de contagion à caractère épidémique). L'état de santé des Montréalais au tournant du siècle mérite d'autant plus d'être étudié que les taux de mortalité infantile des Canadiens français comptaient parmi les plus élevés du monde occidental. Cette exceptionnelle gravité est d'ailleurs attestée par l'intervention gouvernementale. Cette action du gouvernement dans le domaine des «affaires sociales» traditionnellement du ressort des Églises et de la charité publique, devait à coup sûr, être commandée par une situation d'urgence.

Il existe plusieurs manières d'étudier ou de tenter de mesurer l'état de santé d'une population: l'accessibilité aux soins médicaux et hospitaliers; l'étude de la formation des professionnels de la santé, la pratique médicale, la connaissance des conditions sanitaires, l'éducation populaire en matière d'hygiène; voilà parmi d'autres, d'intéressantes voies de recherche. Nous avons choisi de procéder à rebours, en quelque sorte, en étudiant les principales causes de mortalité des Montréalais à la fin du XIXe siècle. Voilà un sujet d'analyse susceptible de jeter quelque lumière sur la question.

\section{LA MORTALITÉ GÉNÉRALE}

Si on jette d'abord un regard global sur les taux de mortalité de Montréal entre 1880 et 1914, on observe des différences pour le moins remarquables (Tableau I). Les quartiers Sainte-Marie et Saint-Jacques, îlots francophones de l'est plafonnent avec des moyennes respectives de 30 et 25 pour 1000 . Le taux de mortalité de Sainte-Marie, par exemple, (quartier ouvrier, francophone et catholique) variait entre 21 et 26 pour 1000 durant la première décennie du XXe siècle après avoir voisiné les 40 pour 1000 pendant les années 1880 . Inutile de souligner le caractère exceptionnellement élevé de ces coefficients de mortalité.

Il est aussi fort éclairant de mettre en relief la dimension géographique de ces différents taux de mortalité. On observe que tous les quartiers situés à l'est du boulevard Saint-Laurent accusent des taux de mortalité supérieurs aux quartiers de l'ouest. La moyenne des taux de mortalité des quartiers francophones de l'est variait aux alentours de 25 pour 1000 alors que celle des quartiers de l'ouest se situerait à près de 15 pour 1000 . Pareilles inégalités méritent d'être soulignées.

Quant à la tendance du taux de mortalité de Montréal entre 1880 et 1914, observons finalement que presque tous les quartiers voient leur coefficient de mortalité chuter dans une proportion notable variant de 30 à 50 pour 100 . Ce phénomène, d'ailleurs 


\begin{tabular}{|c|c|c|c|c|c|c|c|c|c|}
\hline & \multicolumn{9}{|c|}{$\begin{array}{c}\text { Taux de mortalité pour } 1000 \text {, } \\
\text { Montréal selon les quartiers, } \\
1880-1914\end{array}$} \\
\hline$\stackrel{\mathscr{\Xi}}{\stackrel{\Xi}{Z}}$ & 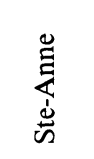 & 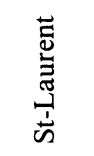 & $\frac{\mathscr{2}}{3}$ & 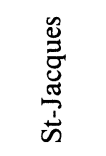 & $\sum_{\substack{5 \\
\infty}}^{\infty}$ & $\begin{array}{l}\text { 岁 } \\
\tilde{\Xi}\end{array}$ & 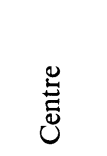 & 虽 & 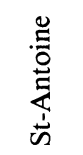 \\
\hline $\begin{array}{l}1880 \\
1881 \\
1882 \\
1883 \\
1884 \\
1885 \\
1886\end{array}$ & $\begin{array}{l}24,25 \\
24,45 \\
23,33 \\
21,75 \\
24,43 \\
28,00\end{array}$ & $\begin{array}{l}17,22 \\
21,64 \\
19,58 \\
18,48 \\
20,73 \\
24,76\end{array}$ & $\begin{array}{l}23,14 \\
24,33 \\
24,01 \\
24,26 \\
23,76 \\
40,75\end{array}$ & $\begin{array}{l}30,48 \\
30,65 \\
29,89 \\
27,63 \\
30,41 \\
61,49\end{array}$ & $\begin{array}{l}29,90 \\
31,24 \\
31,94 \\
32,11 \\
33,85 \\
82,54\end{array}$ & $\begin{array}{r}11,00 \\
12,86 \\
9,29 \\
9,92 \\
10,57 \\
14,40\end{array}$ & $\begin{array}{r}15,00 \\
13,09 \\
18,22 \\
21,32 \\
9,68 \\
32,46\end{array}$ & $\begin{array}{l}24,00 \\
19,58 \\
21,10 \\
23,75 \\
22,48 \\
32,08\end{array}$ & $\begin{array}{l}21,56 \\
18,87 \\
19,84 \\
17,71 \\
18,14 \\
24,04\end{array}$ \\
\hline $\begin{array}{l}1887 \\
1888 \\
1889 \\
1890 \\
1891 \\
1892 \\
1893 \\
1894 \\
1895 \\
1896\end{array}$ & $\begin{array}{l}22,87 \\
21,19 \\
16,57 \\
17,24 \\
16,90 \\
17,21 \\
17,20 \\
15,31 \\
18,13 \\
15,03\end{array}$ & $\begin{array}{l}23,51 \\
23,06 \\
18,21 \\
15,20 \\
15,00 \\
16,10 \\
16,84 \\
16,50 \\
15,67 \\
14,50\end{array}$ & $\begin{array}{l}27,64 \\
28,25 \\
25,04 \\
20,23 \\
22,30 \\
19,37 \\
10,84 \\
22,19 \\
17,23 \\
15,90\end{array}$ & $\begin{array}{l}29,56 \\
31,50 \\
29,52 \\
27,14 \\
26,40 \\
26,09 \\
26,28 \\
29,73 \\
26,84 \\
21,52\end{array}$ & $\begin{array}{l}35,76 \\
41,75 \\
40,14 \\
34,20 \\
31,30 \\
29,03 \\
30,19 \\
36,46 \\
33,29 \\
26,63\end{array}$ & $\begin{array}{r}5,59 \\
5,44 \\
4,30 \\
2,09 \\
15,70 \\
25,88 \\
9,30 \\
4,51 \\
11,40 \\
9,38\end{array}$ & $\begin{array}{r}20,03 \\
16,68 \\
9,62 \\
12,57 \\
19,10 \\
28,57 \\
11,11 \\
24,25 \\
19,63 \\
20,36\end{array}$ & $\begin{array}{l}18,35 \\
12,11 \\
19,32 \\
16,63 \\
23,90 \\
19,63 \\
22,05 \\
19,56 \\
16,94 \\
18,95\end{array}$ & $\begin{array}{l}18,64 \\
16,09 \\
14,81 \\
14,40 \\
15,40 \\
17,81 \\
17,94 \\
16,97 \\
16,83 \\
14,06\end{array}$ \\
\hline $\begin{array}{l}1897 \\
1898 \\
1899 \\
1900 \\
1901 \\
1902 \\
1903\end{array}$ & $\begin{array}{l}11,96 \\
12,53 \\
13,45 \\
11,07 \\
18,53\end{array}$ & $\begin{array}{l}11,05 \\
10,86 \\
13,22 \\
13,55 \\
11,30\end{array}$ & $\begin{array}{l}13,88 \\
15,90 \\
16,93 \\
14,25 \\
15,71\end{array}$ & $\begin{array}{l}21,08 \\
22,28 \\
22,90 \\
19,81 \\
19,61\end{array}$ & $\begin{array}{l}23,29 \\
26,22 \\
27,56 \\
22,57 \\
24,33\end{array}$ & $\begin{array}{l}4,82 \\
7,80 \\
3,03 \\
1,47 \\
8,79\end{array}$ & $\begin{array}{l}23,98 \\
20,95 \\
10,17 \\
15,35 \\
10,57\end{array}$ & $\begin{array}{r}8,22 \\
12,77 \\
9,52 \\
11,61 \\
19,82\end{array}$ & $\begin{array}{l}11,99 \\
13,27 \\
11,84 \\
11,74 \\
12,57\end{array}$ \\
\hline $\begin{array}{l}1904 \\
1905 \\
1906 \\
1907 \\
1908 \\
1909 \\
1910 \\
1911\end{array}$ & $\begin{array}{l}19,66 \\
16,78 \\
16,20 \\
21,68 \\
19,54 \\
21,27\end{array}$ & $\begin{array}{l}13,45 \\
12,44 \\
13,62 \\
13,73 \\
13,87 \\
14,96\end{array}$ & $\begin{array}{l}15,82 \\
17,58 \\
15,72 \\
19,86 \\
17,86 \\
20,53\end{array}$ & $\begin{array}{l}19,60 \\
19,92 \\
18,62 \\
23,89 \\
20,81 \\
23,17\end{array}$ & $\begin{array}{l}27,30 \\
28,38 \\
24,65 \\
25,25 \\
24,40 \\
22,13\end{array}$ & $\begin{array}{l}24,19 \\
11,76 \\
17,54 \\
40,00 \\
26,66 \\
23,33\end{array}$ & $\begin{array}{r}12,09 \\
14,09 \\
7,01 \\
5,89 \\
6,66 \\
8,66\end{array}$ & $\begin{array}{l}19,17 \\
22,93 \\
28,42 \\
31,81 \\
26,95 \\
26,08\end{array}$ & $\begin{array}{l}14,39 \\
14,08\end{array}$ \\
\hline $\begin{array}{l}1912 \\
1913 \\
1914\end{array}$ & $\begin{array}{l}17,20 \\
18,86 \\
17,25\end{array}$ & $\begin{array}{l}14,05 \\
13,28 \\
12,52\end{array}$ & $\begin{array}{l}13,83 \\
14,72 \\
14,87\end{array}$ & $\begin{array}{l}15,62 \\
17,37 \\
16,98\end{array}$ & $\begin{array}{l}22,71 \\
24,34 \\
21,38\end{array}$ & $\begin{array}{l}34,48 \\
12,76 \\
34,04\end{array}$ & $\begin{array}{l}25,47 \\
29,47 \\
35,79\end{array}$ & $\begin{array}{l}15,54 \\
14,86 \\
12,60\end{array}$ & \\
\hline
\end{tabular}




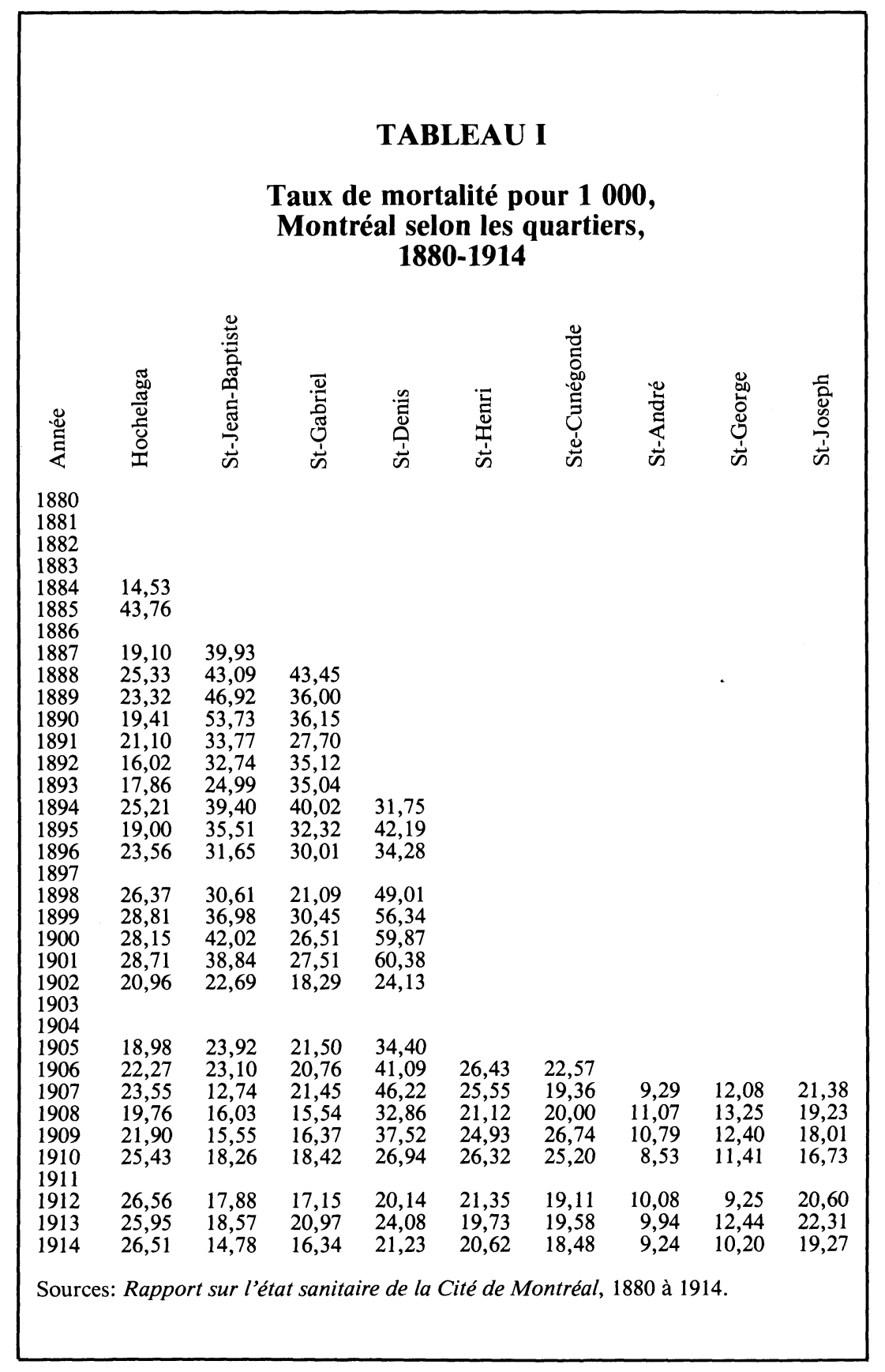


commun à l'ensemble des pays du monde occidental pendant la seconde moitié du XIXe siècle s'explique par la baisse accentuée de la mortalité par maladies contagieuses: principalement la tuberculose, le typhus, la typhoïde et la variole ${ }^{2}$. Nous y reviendrons.

\section{La mortalité infantile}

Les contemporains avaient eux-mêmes observé ${ }^{3}$ que les taux de mortalité infantile de Montréal au tournant du siècle, spécialement chez les Canadiens français, étaient proprement catastrophiques (Tableau II). Exception faite de 1885 où une épidémie variolique s'est abattue sur la métropole, les taux oscillent entre 200 et 290 pour 1000 . Quasi invariablement, les taux de mortalité infantile des anglophones catholiques (Irlandais) sont inférieurs à ceux enregistrés par les catholiques francophones. Enfin, les protestants enregistrent les taux de mortalité infantile les plus bas. Et encore, les derniers taux que nous avons établis ne peuvent être considérés comme tout à fait exacts, car l'enregistrement des naissances chez les protestants ne se faisait pas toujours durant la première année suivant la naissance ${ }^{4}$. C'est donc dire que les taux de mortalité

2 G.B. Longstaff, «The recent decline in the English death-rate considered in connection with the cause of death", Journal of Statistical Society, XLVII (1884): 221-258; S. Phillips, "A review of mortality statistics during the last half century», Clinical Journal, XXX (1908): 55, 61, 73 et 80; A. H. Gale, «Variations in the mortality and incidence of the common infectious diseases of childhood over a century», Proceedings of the Royal Society of Medicine, XXXVI (1943): 97-103; les auteurs précédents sont cités par Th. McKeown et R. G. Record, «Reasons for the Decline of Mortality in England and Wales during the Nineteenth Century», Population Studies, 16, 12 (septembre 1962): 95-96; ainsi que George J. Stolnitz, «A Century of International Mortality Trends», Population Studies, 9, 1 (juillet 1955): $24-55$ et 10,1 (juillet 1956): $17-42$ et W. P. D. Logan, "Mortality in England and Wales from 1848 to 1947», Population Studies, 4,2 (septembre 1950): 132-178.

3 Herbert Brown Ames, The City below the Hill (Montréal, 1896), 85.

4 Voir le Rapport sur l'état sanitaire de la Cité de Montréal (désormais: RESCM) (1877): 6; (1887): 69; (1896): 10. Terry Copp soulève le problème in The Anatomy of Poverty (Toronto, 1974), 96. Les données quantitatives fournies par les RESCM sont de deux ordres: les unes démographiques, les autres, épidémiologiques. Les données démographiques qui nous concernent directement sont le nombre des décès et celui des naissances annuelles; les données épidémiologiques qui apparaissent dans les mêmes rapports concernent surtout les cas de déclaration des maladies contagieuses. Le Bureau de santé de Montréal calculait ses taux de mortalité d'après les listes hebdomadaires des décès qu'étaient légalement tenus de leur fournir les administrateurs des cimetières catholiques, protestants et juifs de la ville. Si l'enregistrement des décès n'offre pas de difficultés majeures, l'enregistrement des naissances, par contre, soulève quelques problèmes. Conformément à la loi civile, le clergé de chaque district de la Province de Québec était tenu, depuis 1826, d'expédier annuellement au protonotaire du district le relevé des naissances. Si l'enregistrement des naissances catholiques n'offre pas de difficultés particulières, il semble que l'enregistrement des naissances protestantes ait été défectueux. A plusieurs occasions les rédacteurs des $R E S C M$ déploreront le fait que «les églises protestantes et juives n'ont pas fait le retour des extraits de baptême (sic)», (1887): 6. Quant aux données épidémiologiques, nous les avons soumises au calcul de deux taux, l'attaque et la léthalité, pour quatre maladies contagieuses (diphtérie, scarlatine, typhoïde et rougeole) dans tous les quartiers de la ville. Or, il est apparu qu'une corrélation directe existait entre l'attaque et la léthalité dans tous les quartiers, alors qu'épidémiologiquement, rien ne réunit ces deux taux. C'est dire qu'en fait, le sous-enregistrement des maladies contagieuses a affecté l'ensemble des quartiers. 


\section{TABLEAU II}

Taux de mortalité infantile selon la dénomination religieuse '000, Montréal 1884-1914

\begin{tabular}{|c|c|c|c|}
\hline Année & $\begin{array}{c}\text { Catholiques } \\
\text { francophones }\end{array}$ & $\begin{array}{c}\text { Autres } \\
\text { catholiques }\end{array}$ & Protestants \\
\hline 1884 & 287,1 & 147,4 & 172,7 \\
\hline 1885 & 408,9 & 189,5 & 198,3 \\
\hline 1886 & 246,9 & 132,1 & 182,7 \\
\hline 1887 & 260,5 & 203,7 & 220,4 \\
\hline 1888 & 279,9 & 241,9 & 236,2 \\
\hline 1889 & 295,8 & 196,8 & 183,7 \\
\hline 1890 & 249,4 & 204,6 & 146,2 \\
\hline 1891 & 262,9 & 211,5 & 160,4 \\
\hline 1892 & 264,3 & 199,0 & 204,3 \\
\hline 1893 & 243,4 & 202,7 & 162,8 \\
\hline 1894 & 249,3 & 222,9 & 177,3 \\
\hline 1895 & 259,0 & 199,6 & 172,3 \\
\hline 1896 & 229,2 & 199,6 & 168,0 \\
\hline 1897 & - & - & - \\
\hline 1898 & 247,5 & 190,5 & 111,7 \\
\hline 1899 & - & - & - \\
\hline 1900 & 282,5 & 235,4 & 102,8 \\
\hline 1901 & 260,4 & 234,3 & 122,9 \\
\hline 1902 & 230,5 & 238,7 & 111,7 \\
\hline 1903 & - & - & - \\
\hline 1904 & - & - & - \\
\hline 1905 & 255,4 & 198,7 & 174,4 \\
\hline 1906 & 252,3 & 198,1 & 153,8 \\
\hline 1907 & - & - & - \\
\hline 1908 & - & - & - \\
\hline 1909 & - & - & - \\
\hline 1910 & - & - & - \\
\hline 1911 & 225,6 & 179,3 & 140,6 \\
\hline 1912 & 200,5 & 179,5 & 123,8 \\
\hline 1913 & 200,3 & 182,3 & 150,2 \\
\hline 1914 & 182,3 & 195,0 & 115,8 \\
\hline
\end{tabular}

Sources: Taux calculés d'après le nombre de naissances vivantes par groupes religieux et le nombre de décès survenus avant l'âge de un an. Données fournies par les RESCM, de 1884 à 1914 . 
infantile que nous avons calculés pour ce groupe ethnique, sont exagérés, dans une proportion invérifiable pour le moment.

Alors que le taux global de mortalité infantile à Montréal variait aux alentours de 260 pour 1000 entre 1896 et 1914, la ville de Toronto enregistrait des taux annuels moyens de 160 pour $1000^{5}$. Songeons qu'à la même époque, la mortalité infantile des soixante-dix-huit plus grandes villes d'Angleterre était aussi d'environ 160 pour $1000^{6}$, alors que la mortalité infantile urbaine en France, entre 1880 et 1897, variait entre 150 et 200 pour $1000^{7}$.

Nous possédons également quelques données concernant les plus grandes villes nord-américaines pour l'année 1910. Observons que Montréal apparaît au troisième rang sitôt après deux villes reconnues pour leur forte population d'origine canadiennefrançaise: Fall River et Lowell... ${ }^{8}$

Comment les contemporains expliquaient-ils cette mortalité infantile si élevée? La mère, le médecin, le laitier et l'État, en prennent tous pour leur compte: on reproche aux mères de priver leurs enfants de l'allaitement maternel; on accuse les producteurs laitiers de vendre du lait impropre à la consommation; on blâme les médecins de ne pas donner suffisamment d'instructions nécessaires aux femmes en couche; et Elzéar Pelletier, secrétaire du Conseil d'hygiène de la province de Québec, terminait son violent plaidoyer à charge en alléguant que «les familles qui n'ont pas fait appeler le médecin auprès de leurs enfants peuvent trop facilement les enfouir sous terre sans être inquiétées pour infraction à l'article $210 \mathrm{du}$ Code criminel» ${ }^{9}$.

Il est même arrivé qu'on impute aux maladies vénériennes un rôle de premier plan au chapitre de la mortalité infantile. Dans un Rapport annuel des officiers de santé de la Cité de Montréal, les docteurs La Roque et Dugdale n'hésitaient pas à déclarer ${ }^{10}$ :

\footnotetext{
5 Terry Copp, The Anatomy of Poverty, 67.

6 A. Daley et B. Benjamin, "London as a case study», Population Studies, 17,3 (mars 1964): 252. Arrivent sensiblement aux mêmes résultats: M. W. Beaver, «Population, Mortality and Milk», Population Studies, 27,2 (juillet 1973): 224; W. O. D. Logan, «Mortality in England and Wales from 1848 to $1947 »$, Population Studies, 4,2 (septembre 1951): 134; Th. McKeown et R. G, Record, «Reasons for the Decline...», 100.

Robert Nadot, «Évolution de la mortalité infantile endogène en France durant la deuxième moitié du XIXe siècle», Population, 25,1 (janvier-février 1970): 55-56. Voir également Maurice Febvay et Marcel Croze, «Nouvelles données sur la mortalité infantile. Influence du milieu social et de la région", Population, 9,3 (juillet-septembre 1954): 390. 8 Liliane Brandt, Facts about Death-rate (New York, New York School of Philanthropy, 1915), 9.

${ }_{9}$ Elzéar Pelletier, Rapport du Conseil d'hygiène de la province de Québec, 19071908, 16. Désormais: $R C H P Q$.

10 Rapport annuel des Officiers de santé de la Cité de Montréal (1872), 7.
} 
D'après les rapports des médecins qui traitent spécialement les maladies vénériennes, et que nous avons consultés, il n'y aurait pas moins de 5,000 cas de ces maladies dans la ville par année.

À l'hôpital général anglais, on traite par année pas moins de 800 cas dont un cinquième sont des maladies syphilitiques constitutionnelles. 200 malades internes parmi lesquels se trouvent un grand nombre d'enfants, subissent des traitements pour différentes maladies syphilitiques constitutionnelles. Nous considérons cette maladie plus désastreuse que les épidémies les plus malignes. Et nous n'hésitons pas à déclarer que la syphilis est une des causes de l'excès de mortalité des enfants.

Les médecins demandent donc aux autorités municipales ${ }^{11}$

l'autorisation de visiter à certaines périodes les maisons de prostitution de la cité, afin de s'enquérir jusqu'à quel degré sévissent les maladies vénériennes (...) pour trouver les moyens efficaces de guérison et de prévention.

Enfin, il ne faut pas négliger l'importance de certains comportements sociaux comme le souligne le docteur Séverin Lachapelle:

Il est une croyance bien grave, dit-il, qui est bien plus répandue et enracinée dans nos familles, qu'elle est partagée par beaucoup de médecins: on croit qu'il est absolument inutile de soigner les maladies des enfants. ${ }^{12}$

Mais de quoi mourait-on? Au chapitre des causes de décès, la tuberculose, à elle seule, s'avère responsable de "plus de un douzième de la totalité des décès» ${ }^{13}$ à la fin du XIXe siècle.

\section{La tuberculose}

La richesse des vocables désignant la tuberculose témoignerait à elle seule de l'importance de cette maladie. Les médecins la diagnostiquaient sous le nom de «phtisie» ou avec encore plus de précision «phtisie pulmonaire»; la bourgeoisie désignait le même mal par «maladie du peuple», «maladie pulmonaire», ou «mal de poitrine»; les esprits plus cultivés évoquaient «la grande fauchause» ou la «peste blanche»; et les classes inférieures (les premières concernées) disaient en parlant d'un tuberculeux, un «consomptif», un «pneumonique», ou encore un «consomption». Peu de maladies se sont mérité autant de dénominations.

11 Ibid., 7.

12 Séverin Lachapelle, La Santé pour tous ou notions élémentaires de physiologie et d'hygiène à l'usage des familles (Montréal, Compagnie d'imprimerie canadienne, 1880), 289.

$13 R C H P Q$ (1895): 35. 
Le caractère éminemment social de la tuberculose est aujourd'hui universellement admis. Après avoir comparé les études françaises, anglaises et américaines sur l'inégalité sociale devant la mort, Jean Daric n'hésite pas à affirmer ${ }^{14}$ :

$\mathrm{Si}$ on isole les décès imputables à la tuberculose pulmonaire, les contrastes entre riches et pauvres s'accentuent de façon si marquée qu'il n'est pas interdit de parler, dans ce cas, de «maladie de classe». Aucune autre cause de décès, et de loin, ne conduit à de telles différences.

Les démographes britanniques McKeown et Record reconnaissent implicitement ce caractère social lorsqu'ils affirment que «the diet is the most significant environmental influence in relation to the trend of mortality from tuberculosis» ${ }^{15}$.

Déjà, en 1910, le docteur S. Lachapelle, nommé à la tête d'une commission royale chargée d'enquêter sur la tuberculose dans la province de Québec, expliquait de la manière suivante l'incidence plus élevée de la tuberculose chez les Canadiens français ${ }^{16}$ :

La population canadienne-française est plus pauvre, elle forme la masse des travailleurs et à cause de cela, est plus exposée aux influences anti-hygiéniques du travail industriel, elle vit dans des habitations moins hygiéniques, sous tous rapports (sic).

Et les Canadiens anglais «occupent les meilleurs emplois, les plus hygiéniques et les plus rémunérateurs, habitent des maisons plus salubres, ont plus souci des applications de l'hygiène». Inutile de dire que ces explications essentiellement ethniques gagneraient largement à être nuancées. Elzéar Pelletier ajoutait en $1901{ }^{17}$ :

Considérant la misère, la pauvreté, les convalescences prolongées de maladies longues et débilitantes, les surmenages physiques prolongés sont très souvent la cause que la consomption fait son oeuvre. (sic)

Concernant la tuberculose dans la province de Québec au début du siècle, nous avons la bonne fortune de bénéficier des conclusions d'une commission royale d'enquête sur la question. L'auteur du rapport établissait que la contribution de la tuberculose à la mortalité générale était de 11 pour $100 \mathrm{chez}$ les populations urbaines et de 9 pour 100 chez les populations rurales et que d'autre part, la proportion des décès par tuberculose pour 100000 de population

\footnotetext{
bre-décembre 1949), 692.

15 Th. McKeown et R. G. Record, «Reasons for the Decline...», 115.

16 Rapport de la Commission royale de la tuberculose [Lachapelle] (1910): 18.

17 RCHPQ (1900-1901): 69.
} 
atteignait 198 en milieu urbain et 163 dans les milieux ruraux. La terrible maladie frappait inégalement les groupes ethniques: la proportion de décès par tuberculose chez les Canadiens anglais se chiffrait à 149 pour 100000 de population alors qu'elle atteignait 201 chez les Canadiens français. Comme unités comparatives, les quartiers montréalais s'avèrent peu probants dans le cas de la tuberculose. L'étude de sections de quartier (délimitées par la densité et le genre d'occupation) serait davantage probante. Signalons cependant la baisse plus qu'appréciable de la mortalité par tuberculose: de 300 pour 100000 de population en 1880 le taux déclinera jusqu'à 180 en $1914^{18}$. Cette chute pourrait s'expliquer par une amélioration des conditions sanitaires minimales ${ }^{19}$.

Outre la tuberculose, il importe de se pencher sur les principales maladies infectieuses qui sévissaient à l'état épidémique ou endémique à Montréal au tournant du siècle. On retiendra les plus meurtrières de celles-ci, soit la variole, la diphtérie, la typhoïde et les maladies diarrhéiques.

\section{La variole}

Ce «mal qui répand la terreur...» et qui a suscité tant de controverses au sujet de la vaccination, a fait l'objet d'une lutte incessante de la part des "officiers» du Bureau de santé de Montréal durant le dernier tiers du XIXe siècle.

Dès 1868 , ceux-ci prenaient nettement position en faveur de la vaccination anti-variolique en admettant que «la vaccination est le seul préservatif contre la petite vérole» ${ }^{20}$. Mais la réticence des citoyens vis-à-vis de la vaccination (l'indifférence du début se transformera en effroi: des émeutiers iront jusqu'à assaillir le Bureau de santé et l'Hôtel de ville en septembre 1885 lors de l'épidémie de variole ${ }^{21}$ ) n'était certainement pas sans motif, comme pourrait le laisser entendre le rapport suivant ${ }^{22}$ :

Mais la vaccination, doit être pratiquée par des personnes compétentes avec du vaccin pur, frais et véritable et non pas avec du pus concrété qui se trouve quelque fois sur le bras après que la vraie gale soit tombée.

$18 \operatorname{RESCM}$ (1914): 29a.

19 Ou encore par des raisons d'ordre nosographique... Autrement dit, l'amélioration du diagnostic aurait-il eu une incidence sur l'évolution réelle de la mortalité par tuberculose? Nous ne pouvons, pour l'instant, évaluer ce phénomène à sa juste mesure.

${ }_{20}$ Rapport des officiers de santé de la Cité de Montréal pour l'année 1868, 1.

21 Il n'est pas superflu de noter que ces événements se produisirent les jours mêmes où le Canada français était secoué par l'affaire Riel.

22 Rapport des officiers de santé de la Cité de Montréal pour l'année 1868, 1. 
Les officiers de santé recommandent donc «en conséquence la vaccination de bras à bras comme la plus sure et la plus efficace» ${ }^{23}$.

Reconnaissons que la méthode jénérienne n'avait rien de très rassurant pour une population du reste nullement informée, et qui nourrissait une suspicion marquée pour la vaccination. Il semble cependant qu'il faille attribuer à l'effort de vaccination antivariolique la chute remarquable des décès par variole après 1885 .

Le Bureau de santé divisait la ville en quatre districts qu'autant de médecins-vaccinateurs se partagaient. Rémunérés 25 dollars par mois, ces médecins devaient consacrer trois heures par jour à la vaccination.

\section{L'épidémie variolique de 1885}

$\mathrm{Au}$ Québec, en 1885, la variole prend soudainement des proportions épidémiques: elle «faucha plus de 5864 existences et en défigurera au-delà de $13000 »{ }^{24}$. À Montréal, la variole se propage avec une virulence et une célérité catastrophiques faisant 3100 victimes.

Certains démographes, tout en reconnaissant l'inégalité sociale devant la mort, semblent prétendre que les épidémies font exception à la règle ${ }^{25}$. Nous croyons qu'au contraire celles-ci peuvent frapper fort inégalement les couches sociales, comme le démontre l'épidémie variolique de Montréal en 1885. L'épidémie n'a pas envahi tous les quartiers avec une égale virulence, loin de là: les quartiers les plus pauvres (Sainte-Marie et Saint-Jacques) sont les plus durement touchés; alors qu'ils représentent en 1885, 27 pour 100 de la population de Montréal, ils récoltent 66 pour 100 des victimes de l'épidémie.

Il est à peine exagéré de dire que l'épidémie de variole a fait ses ravages à l'est du boulevard Saint-Laurent, c'est-à-dire parmi les populations francophones. En effet, ces derniers représentent 91 pour 100 des victimes. Observons finalement que ce sont les enfants qui ont fait les frais de l'épidémie: 80 pour 100 des victimes sont des Canadiens français de moins de 10 ans.

\section{La diphtérie}

À la fin du siècle dernier, les contemporains s'entendent pour déclarer que «la diphtérie est de toutes les maladies contagieuses, celle qui domine le plus dans la province» $(\mathrm{sic})^{26}$. Si nous obser-

\footnotetext{
23 Ibid., 1.

$24 \quad R C H P Q$ (1898-1899): 7.

25 Voir par exemple Roland Pressat, La démographie sociale (Paris, 1971), 43.

26 RCHPQ (1895), 29.
} 
vons l'évolution du taux de décès par diphtérie par 100000 de population à Montréal entre 1880 et 1914, on remarque d'abord une progression quasi géométrique jusqu'en 1887, puis une chute notoire aux alentours de 1890 , et enfin un taux qui se stabilise relativement bas (aux environs de 30 pour 100000 ) pendant la première décennie du XXe siècle. Il faut certainement attribuer à l'utilisation du sérum anti-diphtérique, à partir de 1892, la chute remarquable du taux de décès par diphtérie. L'éradication de cette maladie par l'emploi de l'anatoxine de Ramon ne se réalisera qu'à partir de 1928 au Québec.

\section{La typhoïde}

Telles la dysentrie et les entérites, la typhoïde est d'origine hydrique. C'est-à-dire que l'eau constitue le principal véhicule de cette maladie contagieuse. Comme l'a vérifié Jean-Pierre Goubert pour la ville de Rennes ${ }^{27}$, la purification de l'eau (généralement par l'approvisionnement en eau de source et par la construction d'égouts) annonce le déclin de la typhoïde.

À Montréal, si le taux de mortalité par typhoïde pour 100000 de population accuse une diminution notoire entre 1880 et 1914, il n'en demeure par moins stationnaire entre 1895 et 1914. Nous sommes presque autorisé à avancer que s'il y a eu quelques améliorations au niveau de l'approvisionnement ou du renvoi des eaux usées entre 1885 et 1895 , il semble que l'action municipale (principalement la construction d'égouts) ait ensuite été tout aussi stationnaire que le suggère le taux de décès par typhoïde.

\section{Les maladies diarrhéiques}

À Montréal, à la fin du siècle dernier, l'ensemble des maladies diarrhéiques constitue la principale cause de décès chez les enfants. Mais déjà, on est confronté à une imprécision de taille: sous la rubrique «maladies diarrhéiques» loge un lot de maladies non diagnostiquées dont la diarrhée s'avère n'être qu'un symptôme. On peut prudemment avancer qu'il s'agit principalement de la dysenterie, de la diarrhée et du choléra infantile. Ce qui est certain, c'est qu'il s'agit dans tous les cas de troubles digestifs. C'est donc en partie du côté de l'alimentation et des conditions hygiéniques générales qu'il faudra se tourner pour tenter d'en déceler les causes.

L'évolution du taux de mortalité par maladies diarrhéiques à Montréal connait une forte remontée. Entre 1880 et 1894, le taux

\footnotetext{
27 Jean-Pierre Goubert, «Eaux publiques et démographie historique dans la France urbaine du XIXe siècle. Le cas de Rennes», Annales de démographie historique 1975 (Paris): 115-121.
} 
demeure stationnaire aux environs de 340 pour 100000 de population, mais de 1904 à 1914 on assiste à une nette recrudescence des maladies diarrhéiques, avec des taux se stabilisant autour de 460 pour 100 000. Certains observateurs ont prétendu à bon droit que «la diarrhée eut le triste avantage de remplacer la variole dans les causes de décès d'enfants de moins de un an ${ }^{28}$. À elle seule cette endémie constituait la principale cause de la mortalité infantile à Montréal au début du siècle.

Si nous tentons de regrouper les informations précédentes, un phénomène d'une importance capitale apparaît: c'est celui de la baisse marquée de la mortalité par maladies contagieuses. Tant au niveau de la répartition procentuelle de la mortalité par maladies contagieuses par rapport à la totalité des décès, qu'au niveau des décès par maladies contagieuses pour une population constante de 1880 à 1914, les maladies infectieuses apparaissent de moins en moins dans les registres obituaires. En effet, pendant la première décennie étudiée, 25 à 30 pour 100 des décès annuels étaient attribuables aux maladies contagieuses, alors que durant la dernière décennie à l'étude, ce rapport varie aux environs de 5 à 7 pour 100 . Il faut certainement attribuer cette diminution à l'amélioration des conditions sanitaires générales au tournant du siècle. Mais que savons-nous de cet aspect des «conditions de vie» (concept aussi commode qu'imprécis)?

\section{Le logement}

Il faut le dire tout de suite que de tous les facteurs constituant ce qu'on appelle «conditions de vie», nul n'est indépendant des autres. En 1879, les officiers du Bureau de santé de Montréal établissaient un lien entre surmortalité et logements insalubres: «Le manque d'espace, de lumière et d'air, affirmaient-ils, y est une cause d'insalubrité, aussi constate-t-on dans ces sortes de maison une plus grande mortalité.» ${ }^{29}$

Il semble que l'insalubrité des logements ouvriers montréalais ait fait l'unanimité chez les contemporains. Le docteur J.-I. Desroches écrivait dans son Traité élémentaire d'hygiène privée, $(1888)^{30}$ :

Les ouvriers s'entassent dans les plus pauvres quartiers où les loyers offrent un prix plus accessible à leurs bourses. Ils sont condamnés à vivre pour la plupart dans des logements infects, manquant d'air et d'espace. La plupart du temps, apparte-

28 R. Tanghe, «La population», in Esdras Minville (dir.), Montréal économique (Montréal, 1943), 113.

29 RESCM (1879): 19-20.

30 J.-I. Desroches, Traité élémentaire d'hygiène privée (Montréal, 1888), 132. 
nant à des propriétaires avides de gain et ne connaissant pas la pitié pour le malheureux. Ces habitations ouvrières sont et restent dans les plus mauvaises conditions sanitaires. Aussi, demandez aux médecins dans quelles classes de la société sévissent le plus les maladies contagieuses? Tous vous répondront: dans les familles ouvrières.

Devant les enquêteurs de la Commission royale d'enquête sur les relations entre le capital et le travail au Canada (1888), le docteur Louis Laberge, directeur du Bureau de santé de la ville de Montréal, témoignait de ce que «les maisons des ouvriers en général, pèchent par défaut d'espace», et ajoutait qu' «il y a accumulation générale chez la classe pauvre au point-de-vue des appartements; il n'y a pas de doute sur ce point» ${ }^{31}$.

Les arrivées de plus en plus nombreuses d'immigrants au début du siècle à Montréal semblent avoir aggravé le problème. Les témoignages comme le suivant n'étaient pas exceptionnels ${ }^{32}$ :

Dans la totalité de ces demeures (des immigrants) il y a encombrement; les occupants y souffrent par la négligence des moyens de propreté (...), une grande proportion, sinon la majeure partie sont des colons qui ont à peine quelques ressources, et nous viennent pour la plupart des contrées arriérées de l'Europe, ayant des moeurs et habitudes orientales plutôt qu'européennes, sans la moindre éducation hygiénique. C'est pourquoi ils semblent réfractaires à nos mesures sanitaires; avec cela que la plupart des immigrants ne connaissent souvent ni le français ni l'anglais.

Toutes les études sont là pour le démontrer ${ }^{33}$, qui dit logement insalubre, dit pauvreté. Avant d'invoquer les mentalités et les comportements sociaux, il faut voir sur quoi ils reposent.

\section{L'eau}

Il est inutile d'insister sur l'importance du rôle de l'eau dans l'hygiène publique aussi bien que privée. À Montréal, à la fin du XIXe siècle, l'eau était déjà l'objet d'une taxation. Les usagers qui n'avaient pas honoré leur paiement annuel, trente jours après le 15 août, se voyaient privés d'eau. «Si le locataire ou le propriétaire ne payait pas, la corporation (municipale) poursuivait en justice.» ${ }^{34}$

\footnotetext{
${ }^{31}$ Rapport de la Commission royale d'enquête sur les relations entre le capital et le travail au Canada (Ottawa, 1889), 1: 1. Témoignage du docteur L. Laberge.

32 RESCM (1912): xviii.

33 Voir spécialement Adéline Daumard, «Quelques remarques sur le logement des Parisiens au XIXe siècle», in Annales de démographie historique 1975: 49-64.

34 Rapport de la Commission royale d'enquête sur les relations entre le capital et le travail au Canada, témoignage de J. D. F. Black, trésorier de la ville de Montréal, 1: 248.
} 
Pendant l'hiver de 1887, le docteur J.-I. Desroches affirme que «(...) 800 familles sont actuellement privées d'eau, faute de paiement de la taxe» ${ }^{35}$. L'année suivante, le docteur Louis Laberge rapportait devant la Commission précitée, que ces interruptions de service «existent passablement sur une grande échelle (sic)» ${ }^{36}$. Le même médecin écrit dans son rapport annuel du Bureau de santé pour l'année 1890: "Il est malheureux que le conseil n'ait pas trouvé jour (sic) d'innover d'autres moyens plus humains de percevoir la taxe d'eau que d'en arrêter l'approvisionnement par ses tuyaux de distribution. ${ }^{37}$ Ajoutons que pour quelques cas de pauvreté extrême, le Bureau de santé pouvait plaider une remise ou un délai de paiement de la taxe d'eau devant le Conseil de ville.

\section{L'alimentation}

Un lien direct et évident relie l'état de santé d'une population donnée à son alimentation. Concernant ce segment de l'histoire sociale, nous ne possédons que quelques renseignements fragmentaires sur les problèmes montréalais les plus criants.

Le docteur Georges Grenier, dans un opuscule (Quelques considérations sur les causes de la mortalité des enfants), note que «beaucoup de gens gavent leurs bébés naissants de nourriture solide. Si l'enfant a le malheur de montrer quelque signe de faiblesse, de pâleur ou de maigreur, on s'empresse de le nourrir de pain, biscuits, soupe et de corn-starch (sic)» ${ }^{38}$.

Un autre médecin, pour avoir vacciné un bébé contre la variole, est traduit devant le Collège des médecins à la suite du décès de l'enfant. On l'interroge: «Quelle était la nourriture de cet enfant que vous avez vacciné? Comme les parents sont pauvres (répondit-il), l'enfant mange comme eux, c'est-à-dire du boeuf, du lard, des patates et du lait.» ${ }^{39}$ Voilà un excellent régime pour un débardeur, mais plus ou moins recommandé pour un nourrisson. Habitudes culturelles? Mentalités? Éducation? Seule la pauvreté des classes populaires peut expliquer ce comportement. Et comme l'écrivait le docteur Desroches: «La nourriture est tellement chère

35 J.-I. Desroches, «Bulletin sanitaire de Montréal», in Journal d'hygiène populaire, 3,15 (janvier 1887): 175 .

\footnotetext{
$1: 3$.

36 Rapport de la Commission royale... (1889) témoignage du docteur L. Laberge,

$37 \operatorname{RESCM}$ (1890): 21.

38 Georges Grenier, Quelques considérations sur les causes de la mortalité des enfants (Montréal, 1871), 12.

39 «La vaccination à Montréal», L'Union médicale du Canada, 3,9 (septembre 1874): 419
} 
et les moyens de l'ouvrier sont si restreints que la famille pauvre achète des aliments de qualité inférieure et avariés. ${ }^{40}$

\section{Le lait}

On connaît la corrélation directe qui existe entre la qualité du lait et l'évolution de la mortalité infantile. Tous les témoignages que nous avons recueillis à propos du lait vendu à Montréal au XIXe siècle sont unanimes: le lait n'était presque jamais pur. En 1879 , le directeur du Bureau de santé n'hésitait pas à écrire: "On se sert pour accroître la gravité spécifique du lait, de craie, d'empois et de cervelle de moutons. Le lait est le plus souvent mélangé d'eau (...) ${ }^{41}$ On accuse les laitiers de ne pas prendre la précaution «(...) de traire leurs vaches malades dans des vaisseaux à part, au point que le lait contiendrait souvent du sang, du pus et des cellules anormales» ${ }^{42}$. Le même auteur écrivait en $1885^{43}$ :

Pour adultérer le lait, les laitiers se servent souvent d'eau de puits, très indigeste à cause des sels qu'elle renferme. Parfois cette eau présente un autre danger beaucoup plus grave: les puits sont souvent creusés trop près des étables et l'eau en est contaminée par les infiltrations des liquides provenant des fumiers.

En 1890, le Conseil municipal vota l'article 22 du règlement 105 au terme duquel «nulle personne ne vendra, ou offrira en vente ou aura en sa possession avec l'intention de vendre (...) du lait qui n'atteint pas la moyenne de $3 \%$ de beurre $(. ..){ }^{44}$

La pasteurisation du lait est certainement la première et la plus fructueuse méthode prophylactique pour l'éradication de la mortalité infantile; elle détruit entre autres les bacilles de la typhoïde, du choléra et de la tuberculose. Selon Terry Copp, seules les laiteries des quartiers de l'ouest anglophone de Montréal vendaient du lait pasteurisé ${ }^{45}$. L'organisation de la distribution du lait pasteurisé à Montréal est d'origine privée. Le chanoine Le Pailleur, curé d'une paroisse ouvrière de Montréal, convoque à son presbytère en juin 1910, les médecins de sa paroisse. Ils fondent la première Association de la «Goutte de lait» dans cette paroisse de l'Enfant-Jésus du Mile End dans le but de dispenser aux jeunes mères des conférences sur les soins à donner aux nouveaux-nés. En mars 1913, on constitua le premier Bureau central de l'Association

\footnotetext{
40 J.-I. Desroches, op. cit., 132.

$41 \operatorname{RESCM}$ (1879): 21.

$42 \operatorname{RESCM}$ (1870): 22.

43 RESCM (1885): 100.

44 RESCM (1890): 33.

45 Terry Copp, op. cit., 96.
} 
des «Gouttes de lait» sous la présidence du docteur Séverin Lachapelle. À la fin de la même année vingt-six de ces «Gouttes de lait» paroissiales dispensaient du lait pasteurisé. À l'instar de l'initiative privée, le Bureau de santé de Montréal institua une série de conférences sur les soins à donner aux enfants et ainsi que sur la tuberculose en 1914. «137 conférences ont été données dans 37 paroisses et institutions avec une assistance totale de 41645 personnes.» ${ }^{46}$

Ce n'est qu'aux environs de 1910 que le Bureau de santé de Montréal, encore une fois à la remorque de l'initiative privée, commença à faire oeuvre d'éducation en distribuant gratuitement «sur demande et aux personnes qui font la déclaration de naissance, des pamphlets d'instruction sur les soins à donner aux nouveaux-nés» ${ }^{47}$. L'effet bénéfique des «Gouttes de lait» fut considérable aux dires du directeur du Bureau de Santé qui attestait en 1914 que «le taux de mortalité parmi les enfants qui fréquentent ces «Gouttes de lait» n'a été que de $5 \%$ alors que la mortalité des enfants du même âge dans la ville dépasse $19 \%$ » ${ }^{48}$.

Avant de s'attarder à l'aspect public de l'environnement sanitaire, retenons au passage quelques renseignements concernant l'hygiène privée. Nos renseignements dignes de foi, bien que rarissimes, méritent d'être signalés. Soulignons que «dans la plupart des maisons d'ouvriers, il n'y a pas de salle de bain et on comprend parfaitement que les habitants ne peuvent prendre tous les soins de propreté nécessaires» ${ }^{49}$. Les baignoires étaient évidemment inconnues des classes laborieuses: «(...) pour les habitations des familles ouvrières, où les bains rendraient de si grands services, l'installation de ces appareils y fait complètement défaut.» ${ }^{50}$

\section{Égouts, fosses d'aisance et aspects urbains de l'hygiène publique}

En 1879, le secrétaire du Bureau de santé notait que:

Dans les localités les plus encombrées de la ville, un nombre considérable de fosses d'aisance sont seulement boisées en planches et si peu étanches que le contenu s'en épanche sur le sol circonvoisin. Les excréta des personnes affectées de variole, de diphtérie, de fièvres typhoïdes qu'on n'a pas eu le soin de désinfecter et qui sont jetés dans ces fosses peuvent infecter toute une localité. ${ }^{51}$ 
Les commissaires à l'enquête sur les rapports entre le capital et le travail, demandèrent au docteur L. Laberge ${ }^{52}$ :
À quelle distance les fosses d'aisance se trouvent-elles des maisons? La loi est très défectueuse sur ce point (répondit l'officier de santé) la loi déclare qu'elles devraient être à trois pieds de la maison du voisin, mais elle ne dit pas à quelle dis- tance elles devraient être de la maison du propriétaire lui- même et il arrive souvent que nous avons une fosse d'aisance près de la demeure du locataire.

Aussi, il n'est pas étonnant de voir une nette corrélation entre les quartiers qui ont le plus de fosses d'aisance et les taux de mortalité. Par exemple, en 1887, 87 pour 100 des familles de Sainte-Marie ont des fosses d'aisance et le quartier enregistre un taux brut de mortalité de 35,7 pour 1000 (le plus élevé de la ville); 35 pour 100 seulement des familles de Saint-Antoine ont des fosses d'aisance (il s'agit du plus faible pourcentage de la ville) et enregistre le taux de mortalité le plus bas à l'intérieur des limites de la ville, soit 18,6 pour $1000^{53}$. Le Bureau de santé de Montréal déploya tous les efforts possibles visant à supprimer les fosses d'aisance, foyers d'infection. Cette suppression, à coup sûr, doit compter au nombre des causes de la baisse de la mortalité générale.

Le système d'égouts de la ville de Montréal, dans certains secteurs de l'est de la Cité, était également cause d'insalubrité. Le docteur A.-B. La Roque pouvait écrire sans ambages: «On sait que notre système d'égouts à Montréal est défectueux. Il n'y a jamais eu de plan dont l'ensemble put assurer un système d'assainissement, et qui aurait dû être fait il y a un demi-siècle.» ${ }^{54}$ Son successeur au Bureau de santé y allait encore plus âprement en 188755:

Jusqu'à ces dernières années qu'a-t-on fait à Montréal si ce n'est de creuser et de construire à la hâte 100 verges d'égouts par-ci, 100 verges d'égouts par là, sans s'occuper souvent, si leurs dispositions, seraient assez profondes pour égoutter les caves sur leurs parcours, (...) et puis le problème de leurs raccordements ou leurs connexions futures (sic) y avait-on même pensé? C'est à peine si nous avons 90 milles d'égouts, tandis que la ville (compte) 150 milles de rues.

M. F.-B. Lavallée, inspecteur de la ville, membre d'un comité de trois ingénieurs appointés (sic) pour étudier et faire rapport sur la question des améliorations qui pourraient être faites dans le système régulier de canalisation, mais seulement des sections d'égouts séparées et détachées les unes des autres.

52 Rapport de la Comission royale... (1889), témoignage de Louis Laberge, 1: 3.

53 Pourcentages établis selon les données fournies par le RESCM (1887): 18 et 79.

$\operatorname{RESCM}$ (1879): 23

55 RESCM (1889): 22-23. 
(...) il y a dans la ville 100 milles d'égoûts et à peu près 150 milles de rues, ce qui laisse un tiers de la ville sans canaux. Sur les 100 milles qui existent, il y a à peu près 30 milles qui sont de vieux canaux mal construits.

Toujours au niveau de l'organisation sanitaire municipale en 1885: "Que dire des urinoirs publics? Ils n'existent pas!» ${ }^{56}$ Le premier édicule public à Montréal sera construit en 1912. «La construction d'un chalet de nécessité au Square Jacques Cartier, le premier du genre en notre Cité, sera une innovation avantageuse au point-devue de l'hygiène et de la morale. $)^{57}$ Sur la rue, les passants s'abreuvaient aux fontaines publiques à l'aide de gobelets (retenus par une courte chaîne): "susceptibles d'être le véhicule et le transmetteur des maladies contagieuses et d'aspect malpropre. ${ }^{58}$

Les rues et ruelles des quartiers populaires n'offraient pas un spectacle particulièrement luisant comme le laisse imaginer le rapport du Bureau de santé de $1895^{59}$ :

Une des causes de la malpropreté des ruelles (...) c'est les chiffonniers. Ces gens en quête de quelque chose pour leur subsistance vont continuellement fouiller dans les réceptacles contenant les déchêts (...) non seulement ils fouillent, mais ils renversent les barils, et après le triage, laissent tout à terre. C'est une véritable plaie.

Il semble que le phénomène soit important puisque: «De nombreuses plaintes ont été reçues concernant le vol de réceptacles (...) une classe d'individus les volent et les brisent pour s'en faire du bois de chauffage. ${ }^{60}$ Outre la malpropreté des rues, la présence "dans la ville d'environ 500 étables où l'on y garde des vaches $(. ..){ }^{61}$ n'avait rien pour assainir Montréal. Encore une fois, le partage des «risques» est toujours aussi inégal. C'est le quartier ouvrier SainteMarie, peut-être le plus pauvre de la ville, et qui enregistre les taux de mortalité les plus élevés, qui possède le plus de vaches. Ce dernier aspect est loin d'être négligeable lorsqu'on sait que les vaches constituaient l'un des principaux véhicules du bacille de la tuberculose. À preuve, la chute remarquable de la mortalité infantile lorsqu'on rendit la tuberculinisation des vaches obligatoire au Québec en 1927.

Notons finalement que certains établissements industriels, telles les manufactures de suif, de savon et de charbon animal,

\footnotetext{
$56 \operatorname{RESCM}$ (1885): 97.

RESCM (1912): xviii.

RESCM (1912): xviii.

$\operatorname{RESCM}$ (1895): 33.

RESCM (1895): 33.

61 RESCM (1889): 14.
} 
étaient réputés pour être particulièrement malsaines. Le Bureau de santé était habilité à poursuivre ces fabriques en justice, mais ces dernières, en plus d'invoquer leur participation au bien-être général de la population en fournissant de nombreux emplois et en stimulant le commerce, tergiversaient avec des arguties autour du degré d'insalubrité; ce qui rendait le règlement municipal (luimême imprécis) quasi inopérant ${ }^{62}$.

L'exemple montréalais de 1880 à la Grande Guerre illustre bien l'inégalité sociale devant la mort. Qu'on aborde la question par un biais géo-économique tel le quartier, ou selon des biais culturels telles la dénomination religieuse, l'origine ethnique ou la langue d'usage, une constante se dégage de notre analyse: les Canadiens français de Montréal payent un tribut à la mort considérablement plus élevé que les autres groupes ethniques; invariablement ils disparaissent plus jeunes, et principalement par suite de maladies contagieuses. 